\title{
A 500-kiloton airburst over Chelyabinsk and an enhanced hazard from small impactors
}

\author{
P. G. Brown ${ }^{1,2}$, J. D. Assink ${ }^{3}$, L. Astiz ${ }^{4}$, R. Blaauw ${ }^{5}$, M. B. Boslough ${ }^{6}$, J. Borovička ${ }^{7}$, N. Brachet ${ }^{3}$, D. Brown $^{8}$, M. Campbell-Brown $^{1}$, \\ L. Ceranna ${ }^{9}$, W. Cooke $^{10}$, C. de Groot-Hedlin ${ }^{4}$, D. P. Drob ${ }^{11}$, W. Edwards ${ }^{12}$, L. G. Evers ${ }^{13,14}$, M. Garces ${ }^{15}$, J. Gill ${ }^{1}$, M. Hedlin ${ }^{4}$, \\ A. Kingery ${ }^{16}$, G. Laske ${ }^{4}$, A. Le Pichon ${ }^{3}$, P. Mialle ${ }^{8}$, D. E. Moser ${ }^{5}$, A. Saffer ${ }^{10}$, E. Silber ${ }^{1}$, P. Smets ${ }^{13,14}$, R. E. Spalding ${ }^{6}$, P. Spurný ${ }^{7}$, \\ E. Tagliaferri ${ }^{17}$, D. Uren ${ }^{1}$, R. J. Weryk ${ }^{1}$, R. Whitaker ${ }^{18}$ \& Z. Krzeminski ${ }^{1}$
}

Most large (over a kilometre in diameter) near-Earth asteroids are now known, but recognition that airbursts (or fireballs resulting from nuclear-weapon-sized detonations of meteoroids in the atmosphere) have the potential to do greater damage ${ }^{1}$ than previously thought has shifted an increasing portion of the residual impact risk (the risk of impact from an unknown object) to smaller objects $^{2}$. Above the threshold size of impactor at which the atmosphere absorbs sufficient energy to prevent a ground impact, most of the damage is thought to be caused by the airburst shock wave ${ }^{3}$, but owing to lack of observations this is uncertain ${ }^{4,5}$. Here we report an analysis of the damage from the airburst of an asteroid about 19 metres (17 to 20 metres) in diameter southeast of Chelyabinsk, Russia, on 15 February 2013, estimated to have an energy equivalent of approximately $500( \pm 100)$ kilotons of trinitrotoluene (TNT, where 1 kiloton of TNT $=4.185 \times 10^{12}$ joules). We show that a widely

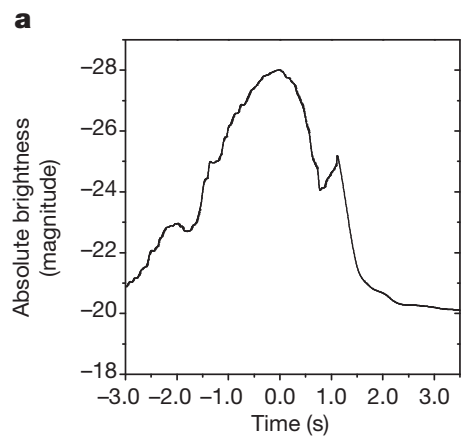

Figure $1 \mid$ Light curve of the Chelyabinsk airburst. a, The brightness profile for the Chelyabinsk airburst, based on indirect illumination measured from video records. The brightness is an average derived from indirect scattered sky brightness from six videos proximal to the airburst, corrected for the sensor gamma setting, autogain, range and airmass extinction, following the procedure used for other airburst light curves generated from video ${ }^{24,25}$. The light curve has been normalized using the US government sensor data peak brightness value of $2.7 \times 10^{13} \mathrm{~W} \mathrm{sr}^{-1}$, corresponding to an absolute astronomical magnitude of -28 in the silicon bandpass. The individual video light curves deviate by less than one magnitude between times -2 and +1.5 with larger deviations outside this interval. Time zero corresponds to 03:20:32.2 UTC on 15 February 2013. b, The energy deposition per unit height referenced technique ${ }^{4-6}$ of estimating airburst damage does not reproduce the observations, and that the mathematical relations ${ }^{7}$ based on the effects of nuclear weapons-almost always used with this technique - overestimate blast damage. This suggests that earlier damage estimates ${ }^{5,6}$ near the threshold impactor size are too high. We performed a global survey of airbursts of a kiloton or more (including Chelyabinsk), and find that the number of impactors with diameters of tens of metres may be an order of magnitude higher than estimates based on other techniques ${ }^{8,9}$. This suggests a non-equilibrium (if the population were in a long-term collisional steady state the size-frequency distribution would either follow a single power law or there must be a size-dependent bias in other surveys) in the near-Earth asteroid population for objects 10 to 50 metres in diameter, and shifts more of the residual impact risk to these sizes.

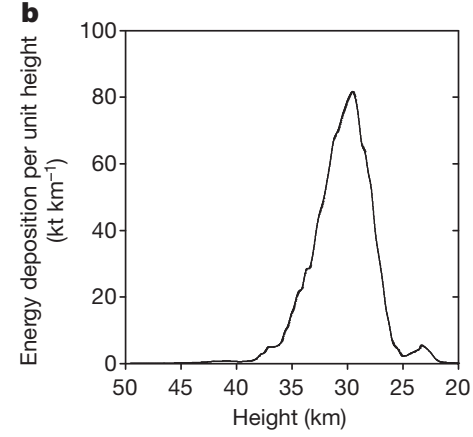

for the Chelyabinsk airburst, based on video data. The conversion to absolute energy deposition per unit path length assumes a blackbody emission of $6,000 \mathrm{~K}$ and bolometric efficiency of $17 \%$, the same as the assumptions used to convert earlier US government sensor information to energy ${ }^{26}$. The heights are computed using the calibrated trajectory ${ }^{10}$ and features of the light curves common to different video sites, resulting in a height accuracy of about $1 \mathrm{~km}$. The total energy of the airburst found by integrating under the curve exceeds $470 \mathrm{kt}$. The half-energy-deposition height range is $33-27 \mathrm{~km}$; these are the heights at which energy deposition falls below half the peak value of approximately $80 \mathrm{kt}$ per kilometre of height, which is reached at an altitude near $29.5 \mathrm{~km}$.

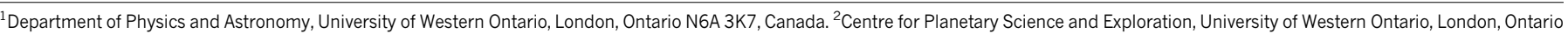

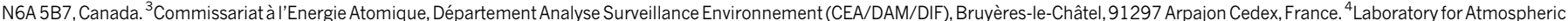

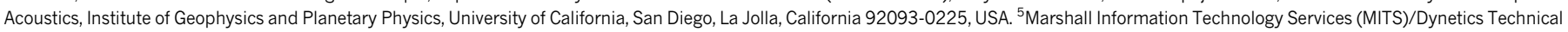

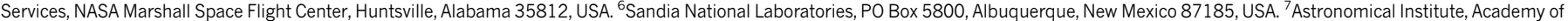

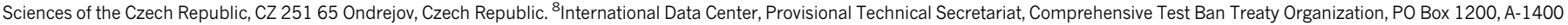

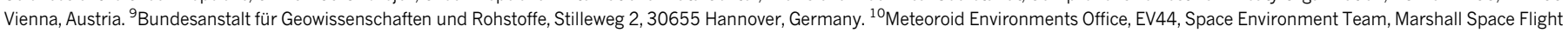
Center, Huntsville, Alabama 35812, USA. ${ }^{11}$ Space Science Division, Naval Research Laboratory, 4555 Overlook Avenue, Washington DC 20375, USA. ${ }^{12}$ Natural Resources Canada, Canadian Hazard

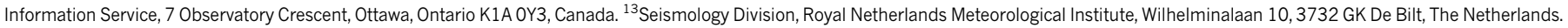
${ }^{14}$ Department of Geoscience and Engineering, Faculty of Civil Engineering and Geosciences, Delft University of Technology, Stevinweg 1,2628 CN Delft, The Netherlands. ${ }^{15}$ Infrasound Laboratory,

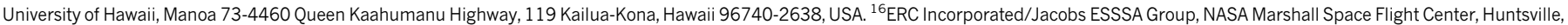

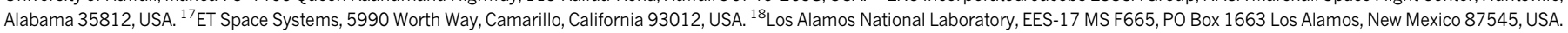


The Chelyabinsk airburst ${ }^{10}$ was observed globally by multiple instruments-including infrasound, seismic, US government sensors and more than 400 video cameras-at ranges up to $700 \mathrm{~km}$ away. The resulting airblast (shock wave travelling through the air from an explosion) shattered thousands of windows in urban Chelyabinsk, with flying glass injuring many residents.

Data from US government sensors timed the peak brightness to 03:20:32.2 UTC (coordinated universal time) on 15 February 2013 with an integrated radiated energy of $3.75 \times 10^{14} \mathrm{~J}$ and a peak brightness of $2.7 \times 10^{13} \mathrm{~W} \mathrm{sr}^{-1}$. These values correspond to an estimated energy equivalent of about $530 \mathrm{kt}$ of TNT. The peak brightness was equivalent to an absolute astronomical magnitude of -28 (referenced to a range of $100 \mathrm{~km}$ ) in the silicon bandpass, making the airburst appear 30 times brighter than the Sun to an observer directly under this point. The airburst's light curve has been reconstructed by considering the measured light production from several video records (see Supplementary Information for details) as shown in Fig. 1. We note that point-like models $s^{4-6}$ of airburst energy deposition, which treat the impactor as a strengthless, liquid-like material, predict that the height range in which the energy deposition per unit path length falls to half its maximum value is less than $2 \mathrm{~km}$ for impacts as shallow $\left(17^{\circ} \text { from the horizontal }\right)^{10}$
Table 1 | Energy estimates for the Chelyabinsk airburst

\begin{tabular}{lll}
\hline Technique & Best estimate (kt) & Range (kt) \\
\hline Seismic & 430 & $220-630$ \\
Infrasound (mean period) & 600 & $350-990$ \\
US government sensor & 530 & $450-640$ \\
Video-derived lightcurve & $>470$ & \\
\hline
\end{tabular}

Here 'kiloton' refers to the energy equivalent to a kiloton of TNT. To estimate the energy from infrasonic airwaves, all 42 infrasound stations of the International Monitoring System ${ }^{23}$ were examined. Of these, 20 stations showed clear signals from the airburst. Our infrasound energy estimates are based on the average observed dominant infrasound period from 12 stations that have stratospheric returns showing the highest signal-to-noise ratio. Seismic Rayleigh waves generated by the airburst shock wave impinging on the Earth's surface just south of Chelyabinsk were detected by about 70 seismic stations at ranges in excess of $4,000 \mathrm{~km}$. The amplitude of these waves in specific passbands as calibrated to nuclear airbursts ${ }^{19}$ were used as an independent estimate of source energy.

as that of Chelyabinsk, which is less than one-third of the observed value (more than $6 \mathrm{~km}$ ). (We note that any object striking the Earth or its atmosphere is an impactor; a ground impactor creates a crater, but most burn up before that, releasing a large amount of energy into the atmosphere as an airburst.) Airburst energy estimates from four different techniques are summarized in Table 1. Our preferred mean energy estimate is in the range of $400-600 \mathrm{kt}$. Details of the analysis
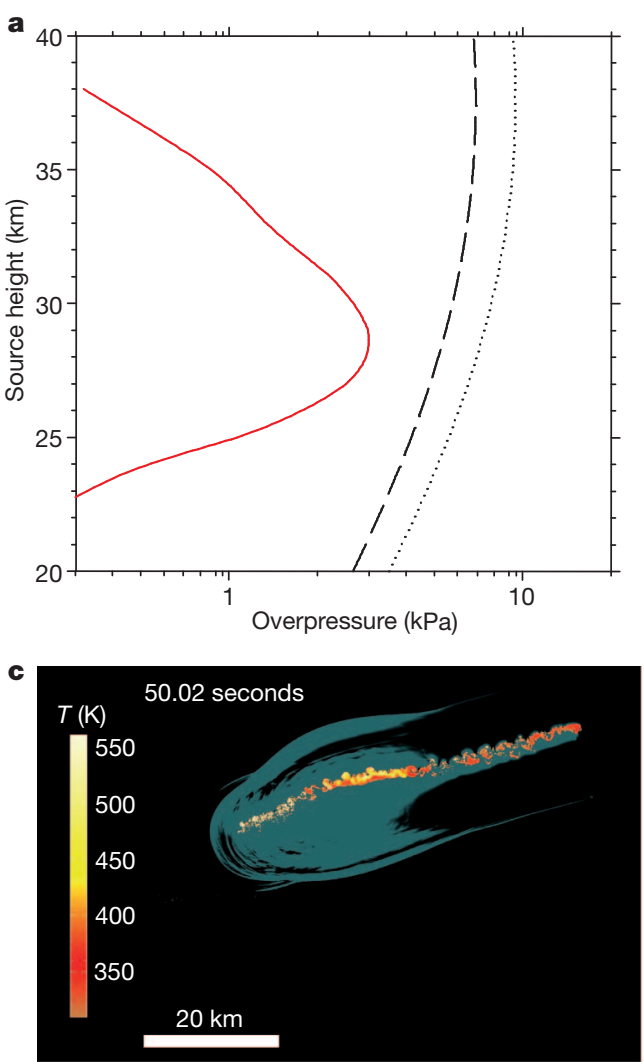

Figure $2 \mid$ Observed and predicted shock characteristics for the Chelyabinsk airburst. a, Theoretical airblast overpressures using standard nuclear weapons relations $s^{7}$ and the cylindrical-line source blast theory ${ }^{13}$ (which assumes the explosion occurs so swiftly that it is equivalent to a single instantaneous detonation of a long cylindrical line of explosive charge) that is appropriate to central Chelyabinsk. The nuclear relation curves (in black) assume a spherical point source at a specific height and show assumed yields of $500 \mathrm{kt}$ (dashed line) and $1 \mathrm{Mt}$ (dotted line). The cylindrical-line source airblast model (red line) uses the energy deposition per unit length from Fig. $1 \mathrm{~b}$ to define an equivalent blas radius as the source and assumes that the shock is linear at the ground (linear means its amplitude is low enough to be well approximated as moving at the local ambient speed of sound and non-linear effects are negligible).

b, Travel-time residuals between the time of airburst passage at each height and the main airblast arrival for 38 videos (see Supplementary Table 5). The residuals (black circles) show the observed arrival time (corrected for fireball
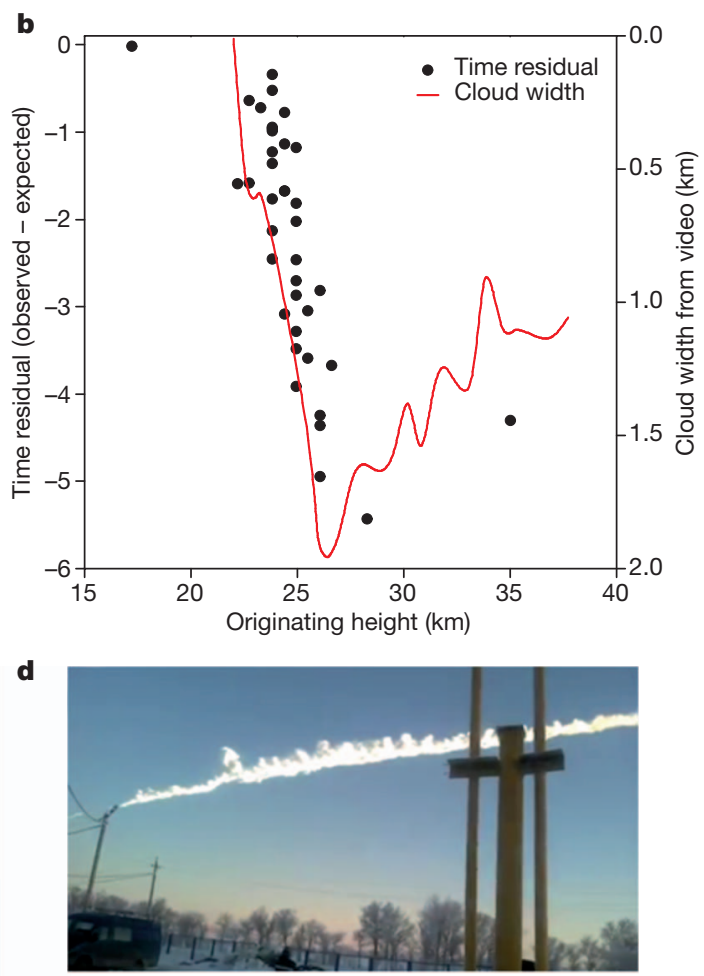

motion) minus the expected time, calculated assuming propagation at the local adiabatic sound speed and incorporating winds ${ }^{11}$. For comparison, the width of the visible cloud trail is shown (red line). This is consistent with the shock wave travelling faster than the ambient sound speed near the airburst. The minimum timing residuals suggest that shock source heights vary between $30 \mathrm{~km}$ and $23 \mathrm{~km}$ across Chelyabinsk, as opposed to originating from a point source. c, Modelling the temperature of the trail (colour scale). The apparent crosssection of CTH simulation $50 \mathrm{~s}$ after $0.5 \mathrm{Mt}$ was released into segmented cylinders of air. The outer blue-grey shaded area reveals an envelope of shocked air-note that the dominant shape of the shock is cylindrical. d, For comparison with c, a video frame of the dust cloud taken $250 \mathrm{~km}$ to the southwest of the airburst path looking North is shown (http://www.youtube.com/ watch? $\mathrm{v}=\mathrm{lCv} 9 \mathrm{~S} 0 \mathrm{Z0} 0 \mathrm{eE}$, taken by E. Volkov). Approximately $140 \mathrm{~km}$ of the end portion of the airburst trail is shown, some $40-60 \mathrm{~s}$ after the passage of the fireball. 
procedures and measurements are given in the Supplementary Information.

To establish the nature and source height of the airblast that caused damage in Chelyabinsk, we used the known trajectory ${ }^{10}$ and a suite of videos (see Supplementary Table 5) that recorded both the airburst and the main airblast arrival. We computed acoustic travel times from each point on the airburst trajectory to each video location using a propagation model including winds that was developed for earlier airburst infrasound analyses ${ }^{11}$. The results show that the first airblast wave (which also produced the damage) arrived from different altitudes at different sites, consistent with a cylindrical shock from the extended airburst, as opposed to a more point-like explosion. The timing residuals between the observed and expected arrivals follow the bolide trail size, as shown in Fig. 2b, consistent with the shock being strong early in its propagation. The airblast reaching the city of Chelyabinsk was generated at altitudes of $24-30 \mathrm{~km}$, roughly from the peak to the end of the main airburst.

In Fig. 2a we show overpressure predictions from standard airblast relations based on nuclear explosions ${ }^{7}$, as used by most impact-effect models $\mathrm{s}^{4,6,12}$. For comparison, the predictions of cylindrical-line source airblast theory applied to meteor entry ${ }^{13}$ are also shown. The airblast overpressure in Chelyabinsk from window breakage measurements is $3.2 \pm 0.6 \mathrm{kPa}$ (see Supplementary Information for details). We note the overestimation of overpressure using the nuclear blast relations ${ }^{7}$, an effect others have suggested in connection with airbursts ${ }^{4}$. Given that nuclear explosions release half their energy as radiation ${ }^{7}$, thus reducing the effective yield of airblast energy, the nuclear curve in Fig. 2a that is most appropriate to Chelyabinsk is about $1 \mathrm{Mt}$.

To examine whether a fragmentation model ${ }^{14}$ is consistent with the observed data and estimated object size, we have applied an entry code based on a progressive fragmentation model of the initial object. Assuming an initial meteoroid of diameter $19 \mathrm{~m}$ and a tensile strength at first fragmentation of $0.7 \mathrm{MPa}$ (ref. 10), with ablation ending at about $27 \mathrm{~km}$ once most of the energy has been lost, we find a reasonable match to both the light curve and early dynamics. The final main fragmentations in this model occur near $4 \mathrm{MPa}$, very similar to those observed $(1-5 \mathrm{MPa})$ in the most severe fragmentation portion of the airburst ${ }^{10}$. The dynamics and light production from the model are not realistic near the terminal phase of the airburst because the model assumes that all fragments split identically at each fragmentation epoch. This is in contrast to observations at the end of the airburst where one leading fragment was observed ${ }^{10}$ (as opposed to dozens of identically sized individuals).

To further define the nature of the shock, we have used the well known CTH simulation framework used for the Tunguska ${ }^{15}$ airburst and impactors ${ }^{1}$ comparable to the asteroid causing the Chelyabinsk airburst. The simulation used all the observed trajectory parameters ${ }^{10}$ and the observed energy as a function of height (Fig. 1b) to mimic the entry process by creating an instantaneous energy release in a sequence of momentum-preserving air cylinders along the airburst path, scaled such that the total integrated energy is $500 \mathrm{kt}$. Figure $2 \mathrm{c}$ and $\mathrm{d}$ shows the result of this simulation and comparison to a video record of the dust cloud generated by the airburst at a similar time. The notable characteristics are that the primary shock is cylindrical, in contrast to point-source energy release airburst models ${ }^{4-6}$, which have a strong spherical shock component. Instabilities that result from fast-rising buoyant air in the simulation produce similar structures to those seen in videos of the dust cloud.

Model overpressures for central Chelyabinsk are found to be $3 \mathrm{kPa}$, consistent with observations. Our estimates of overpressure are based on window breakage (see Supplementary Fig. 5) confined to a small region in Chelyabinsk. The CTH simulations were run for more than three minutes after the airburst, producing model variations of overpressure across the entire city of Chelyabinsk which were smaller than the differences produced by local effects, such as shock reflections from buildings, numerical uncertainty in the simulation and our generally

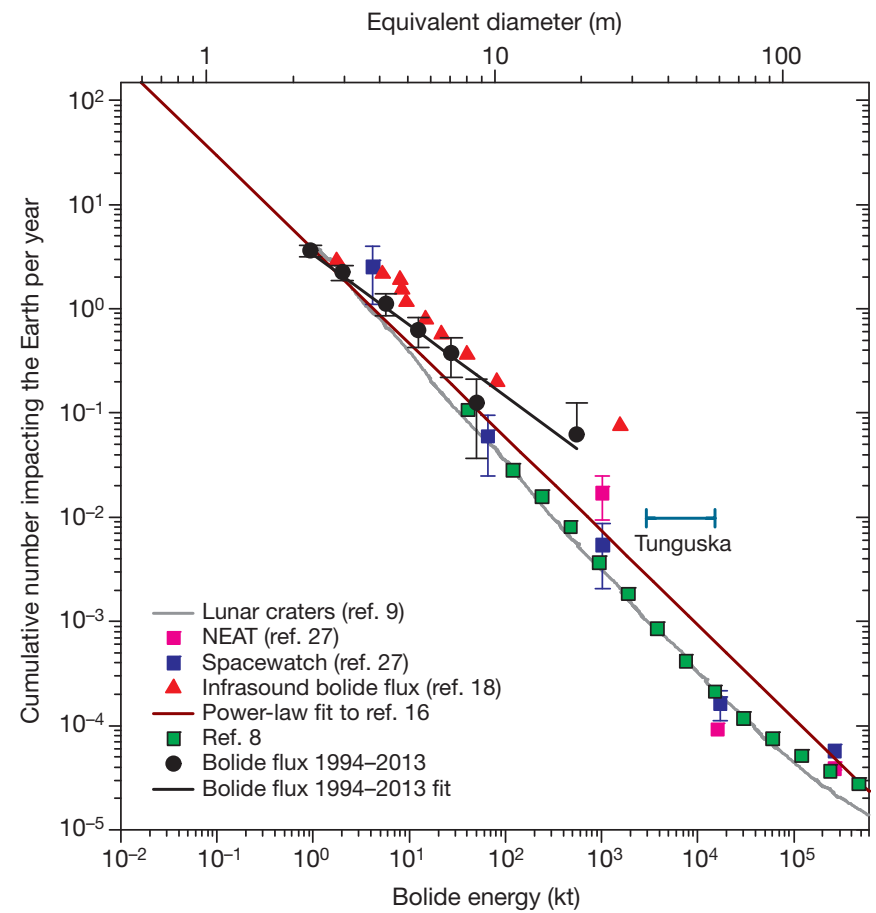

Figure 3 The estimated cumulative flux of impactors at the Earth. The bolide impactor flux at the Earth (bolide flux 1994-2013; black circles) is based on about 20 years of global observations from US government sensors and infrasound airwave data. Global coverage averages $80 \%$ among a total of 58 observed bolides with $E>1 \mathrm{kt}$ and includes the Chelyabinsk bolide (rightmost black circle). This coverage correction is approximate and the bolide flux curve is probably a lower limit. The brown line represents an earlier power-law fit from a smaller data set for bolides $1-8 \mathrm{~m}$ in diameter ${ }^{16}$. Error bars represent counting statistics only. For comparison, we plot de-biased estimates of the near-Earth-asteroid impact frequency based on all asteroid survey telescopic search data until mid-2012 (green squares) ${ }^{8}$ and other earlier independently analysed telescopic data sets ${ }^{27}$ including the NEAT discoveries (pink squares) and the Spacewatch survey (blue squares), where diameters are determined assuming an albedo of 0.1 . From the telescopically determined number of nearEarth asteroids and their typical orbits we can compute the average interval between Earth impactors of a given energy. Energy for telescopic data was computed assuming a mean bulk density of $3,000 \mathrm{~kg} \mathrm{~m}^{-3}$ and average impact velocity of $20.3 \mathrm{~km} \mathrm{~s}^{-1}$. The intrinsic impact frequency for these telescopic data was found using an average probability of impact for near-Earth asteroids of $2 \times 10^{-9}$ per year for the entire population of asteroids. Lunar crater counts converted to equivalent impactor flux and assuming a geometric albedo of 0.25 (grey solid line) are shown for comparison", although we note that contamination by secondary craters and modern estimates of the near-Earthasteroid population that suggest lower albedos will tend to shift this curve to the right and downwards. Finally, we show the estimated influx from global airwave measurements conducted from 1960 to 1974, which detected larger (5-20 m) bolide impactors (red triangles) ${ }^{18}$ using an improved method for energy estimation compared to earlier interpretations of the same data.

small number statistics. This limits our ability to validate the simulated CTH overpressure spatially.

Using our best estimate for the Chelyabinsk airburst energy, of about $500 \mathrm{kt}$, we have estimated the bolide flux at the Earth over the period from 1994 to mid-2013. This estimate is based on 20 years of total global coverage by the US government or infrasound sensors, more than doubling the earlier time coverage ${ }^{16,17}$. All events with estimated yields in excess of $1 \mathrm{kt}$ are included. Figure 3 shows that this bolide flux at small sizes (less than $5 \mathrm{~m}$ in diameter) is in agreement within uncertainties with telescopic ${ }^{8}$ data and earlier infrasonic ${ }^{18}$ influx estimates. However, at larger diameters $(15-30 \mathrm{~m})$, both the bolide and infrasound ${ }^{18}$ flux curves show an apparent impact rate at the Earth an order of magnitude larger than either that estimated by 
telescopic surveys or the longer-term average impact rate provided by lunar cratering. In both cases these deviations well above the constant power-law slope of ref. 16 are due to single large events, so caution must be exercised owing to the small number statistics. A best-fit regression line to the bolide flux is given by $N=a E^{-b}$, where $N$ is the cumulative number of objects with energy $E$ (in kilotons) or more that impact the Earth per year, $a=3.31 \pm 0.11$ and $b=-0.68 \pm 0.06$. We note that excluding the rightmost two points in Fig. 3 (representing Chelyabinsk and two other events larger than $30 \mathrm{kt}$ ) produces a nearly identical slope.

Using the telescopic impact frequency ${ }^{8}$ (green squares in Fig. 3) as a baseline for the 20-year period of the bolide survey, there is only a $13 \%$ chance that any random 20 -year period would have an airburst as large or larger than Chelyabinsk. The independent 14-year survey by infrasound ${ }^{18}(1960-74)$ detected a probable $\sim 1.5$-Mt airburst on 3 August 1963. Such a large event would be expected at the $\sim 3 \%$ level during such a survey period. Although these deviations may be attributable to small number statistics, we note that Tunguska, with a source energy (energy released at the location of the explosion) of the order of 3-15 Mt (refs 15,19; shown as a horizontal line in Fig. 3) is also an extreme outlier (expected at the $2-10 \%$ level to have occurred during the past century). These events, taken together with Chelyabinsk, are increasingly suggestive of non-equilibrium in the impactor flux for near-Earth asteroids that are $10-50 \mathrm{~m}$ in diameter. This is manifested as a change in the power-law energy-frequency distribution at these sizes, similar to changes in the power law at other sizes ${ }^{20}$. This is also consistent with the recent origin of Chelyabinsk as a single near-Earth asteroid and a possible link to asteroid 86039 (ref. 10). Our findings support earlier interpretations of an influx maximum at this size range ${ }^{21,22}$. We note that telescopic surveys have only discovered about 500 near-Earth asteroids that are $10-20 \mathrm{~m}^{\text {in }}$ diameter $^{8}$ (comparable to the Chelyabinsk asteroid) of an estimated near-Earth asteroid population (http://ssd.jpl.nasa.gov) of around $2 \times 10^{7}$, implying that a nonequilibrium impactor population at these sizes could be present but not yet apparent in the discovered near-Earth asteroid population.

Received 27 June; accepted 3 October 2013.

Published online 6 November 2013.

1. Boslough, M. B. \& Crawford, D. A. Low-altitude airbursts and the impact threat. Int. J. Impact Eng. 35, 1441-1448 (2008).

2. Harris, A. W. What Spaceguard did. Nature 453, 1178-1179 (2008)

3. Chapman, C. R. \& Morrison, D. Impacts on the Earth by asteroids and comets: assessing the hazard. Nature 367, 33-40 (1994).

4. Collins, G. S., Melosh, H. J. \& Marcus, R. A. Earth Impact Effects Program: a web-based computer program for calculating the regional environmental consequences of a meteoroid impact on Earth. Meteorit. Planet. Sci. 40, 817-840 (2005).

5. Chyba, C. F., Thomas, P. J. \& Zahnle, K. J. The 1908 Tunguska explosion: atmospheric disruption of a stony asteroid. Nature $\mathbf{3 6 1}, 40-44$ (1993).

6. Hills, J. G. \& Goda, M. P. The fragmentation of small asteroids in the atmosphere. Astron. J. 105, 1114-1144 (1993)

7. Glasstone, S. \& Dolan, P. J. The Effects of Nuclear Weapons 3rd edn, 100-105 (US Gov. Printing Office, 1977)

8. Harris, A. The value of enhanced NEO surveys. IAA-PDC13-05-09 (Planetary Defence Conference, IAA, 2013)

9. Werner, S. C., Harris, A. W., Neukum, G. \& Ivanov, B. A. The near-Earth asteroid sizefrequency distribution: a snapshot of the lunar impactor size-frequency distribution. Icarus 156, 287-290 (2002).
10. Borovička, J. et al. The trajectory, structure and origin of the Chelyabinsk asteroidal impactor. Nature http://dx.doi.org/10.1038/nature12671 (this issue).

11. Brown, P. G, etal. Analysis of a crater-forming meteorite impact in Peru. J. Geophys. Res. 113, 1-13 (2008).

12. Toon, O. B., Zahnle, K. J., Morrison, D., Turco, R. P. \& Covey, C. Environmental perturbations caused by the impacts of asteroids and comets. Rev. Geophys. 35, 41-78 (1997).

13. Revelle, D. O. On meteor-generated infrasound. J. Geophys. Res. 81, 1217-1230 (1976).

14. ReVelle, D. O. in Proc. Int. Astron. Union Symp. No. 236 (NEOs, our Celestial Neighbors: Opportunity and Risk) (eds Milani, A., Valsecchi, G. B. \& Vokrouhlický, D.) 95-106 (Cambridge Univ. Press, 2007).

15. Boslough, M. B. \& Crawford, D. Shoemaker-Levy 9 and plume-forming collisions on Earth. Ann. NY Acad. Sci. 822, 236-282 (1997).

16. Brown, P. G., Spalding, R. E. \& ReVelle, D. O. Tagliaferri, E. \& Worden, S. P. The flux of small near-Earth objects colliding with the Earth. Nature 420, 294-296 (2002).

17. Ens, T. A., Brown, P. G., Edwards, W. N. \& Silber, E. Infrasound production by bolides: a global statistical study. J. Atmos. Sol. Terr. Phys. 80, 208-229 (2012)

18. Silber, E. \& ReVelle, D. O. Brown, P. G. \& Edwards, W. N. An estimate of the terrestria influx of large meteoroids from infrasonic measurements. J. Geophys. Res. 114, E08006 (2009)

19. Ben-Menahem, A. Source parameters of the Siberian explosion of June 30,1908, from analysis and synthesis of seismic signals at four stations. Phys. Earth Planet. Inter. 11, 1-35 (1975).

20. Bland, P. A. \& Artemieva, N. The rate of small impacts on Earth. Meteorit. Planet. Sci. 41, 607-631 (2006)

21. Ceplecha, Z. Influx of interplanetary bodies onto Earth. Astron. Astrophys. 263, 361-366 (1992)

22. Ward, S. \& Asphaug, E. Asteroid impact tsunami: a probabilistic hazard assessment. Icarus 145, 64-78 (2000).

23. Brachet, N etal. in Infrasound Monitoring for Atmospheric Studies (eds Le Pichon, A Blanc, E. \& Hauchecorne, A.) 77-118 (Springer, 2010)

24. Brown, P. G. et al. The fall of the Grimsby meteorite-l: Fireball dynamics and orbit from radar, video, and infrasound records. Meteorit. Planet. Sci. 46, 339-363 (2011).

25. Borovička, J. etal. The Košice meteorite fall: atmospheric trajectory, fragmentation, and orbit. Meteorit. Planet. Sci. http:dx.doi.org/10.1111/maps.12078 (2013).

26. Tagliaferri, E., Spalding, R., Jacobs, C., Worden, S. P. \& Erlich, A. in Hazards Due to Comets and Asteroids (ed. Gehrels, T.) 199-221 (Univ. Arizona Press, 1994).

27. Rabinowitz, D. Helin, E. Lawrence, K. \& Pravdo, S. A reduced estimate of the number of kilometer-sized near-Earth asteroids. Nature 403, 165-166 (2000).

Supplementary Information is available in the online version of the paper.

Acknowledgements Funding was provided by the NASA co-operative agreement NNX11AB76A and the Czech institutional project RVO:67985815. D.P.D. acknowledges support from the Office of Naval Research. We appreciate discussions with F. Gilbert (of UCSD), J. Stevens (of SAIC), P. Earle and J. Bellini (of USGS). D. Dearborn provided assistance with video reductions.

Author Contributions P.G.B., N.B., D.B., L.C., W.E., L.G.E., M.G., A.L.P., J.D.A., P.M., P.Smets and R.W. performed various aspects of the identification, measurement and interpretation of infrasound records. L.A., C.d.G.H., M.H. and G.L. collected and identified the airburst signals in seismic recordings as well as analysing and interpreting the seismic data. P.G.B., R.B., J.B., W.C., J.G., A.K., D.E.M., R.W., A.S. and P. Spurny helped in identifying important videos and their geolocation and various aspects of their measurements. M.B.B. and M.C.-B. performed bolide entry modelling. D.P.D. provided atmospheric model data and interpretation. Z.K., J.G. and R.J.W. performed video lightcurve analysis and calibrations and helped with their interpretation as well as performing measurements of video dust cloud features. R.E.S. and E.T. facilitated and interpreted US Government Sensor data. D.U. performed window breakage analysis. P.G.B. and E.S. performed analysis of acoustic propagation and associated computer code development. P.G.B. wrote the manuscript. All authors commented on the manuscript.

Author Information Reprints and permissions information is available at www.nature.com/reprints. The authors declare no competing financial interests. Readers are welcome to comment on the online version of the paper. Correspondence and requests for materials should be addressed to P.G.B. (pbrown@uwo.ca). 